\title{
High-Frequency EPR Instrumentation
}

\author{
Edward J. Reijerse
}

Received: 6 May 2009/Revised: 16 June 2009/Published online: 15 November 2009

(C) The Author(s) 2009. This article is published with open access at Springerlink.com

\begin{abstract}
An overview of the most recent developments in high-frequency highfield electron paramagnetic resonance (EPR) instrumentation is given. In particular, the practical choices concerning sources, detectors, resonators, propagation systems as well as magnet technology are discussed in the light of various possible applications. Examples of particular homodyne and heterodyne quasi-optic EPR systems illustrate the potential for future developments in EPR technology.
\end{abstract}

\section{Introduction}

In the last few decades, an increasing number of electron paramagnetic resonance (EPR) spectrometers have been developed employing microwave frequencies significantly higher then the "conventional" $\mathrm{X}$ - and Q-band range (i.e., 9 and $35 \mathrm{GHz}$ ). The microwave bridge in these instruments typically uses technology deviating from the traditional waveguides and coaxial lines, and the magnetic field is generated using superconducting magnets. We will (somewhat arbitrarily) define EPR spectrometers operating with frequencies starting from $90 \mathrm{GHz}$ and magnetic fields around $3 \mathrm{~T}$ and upwards as "high-frequency high-field (HF)-EPR spectrometers". The motivation to develop these instruments is usually driven by one of the following experimental requirements.

1. Increased $g$-resolution: As in nuclear magnetic resonance (NMR), the spectral resolution of the Zeeman interaction is proportional to the applied field and frequency. One can only benefit from this effect, however, when the line widths in the EPR spectrum are "field independent". Metalloproteins, in which the line width is dominated by $g$-strain, do not show any improvement in $g$-resolution at higher magnetic fields. In fact, the increased line width may wipe out hyperfine

E. J. Reijerse ( $\square)$

Max-Planck Institut für Bioanorganische Chemie, Mülheim an der Ruhr, Germany

e-mail: reijerse@mpi-muelheim.mpg.de 
structures, thus deteriorating the overall spectral resolution. For radicals in solution and solid state, in general, the line width is dominated by residual hyperfine interaction, which is field independent. Therefore, radical EPR applications benefit particularly from going to higher fields.

2. Higher (Absolute) Sensitivity: As long as it is possible to use single-mode resonators, the cavity volume will scale down with increasing frequency. Consequently, for constant (small) sample size, going to higher frequencies will increase the filling factor of the cavity very rapidly. For non-lossy, nonsaturating samples, the absolute sensitivity theoretically increases proportional to $\omega^{7 / 2}$ at constant incident power. The concentration sensitivity (sample volume scales with cavity volume) should theoretically increase proportionally to $\omega^{1 / 2}[1,2]$. In practice, however, it often proves difficult to approach these theoretical limits.

3. Study of Motional Dynamics: When the movement of paramagnetic species induces spectral changes proportional to the external magnetic field, the dynamics of these movements can be studied best at progressively increasing magnetic fields. Dynamics of which the spectral effect can be characterized as "fast exchange", when studied at low frequency, will change their regime to "slow exchange" when studied at high frequency. A multi-frequency study will enable a full characterization of the kinetic and thermodynamic parameters of such a dynamic process. In particular, the study of spin label dynamics intensely benefits from the multi-high-frequency approach, since here, also the geometrical factors are accessible in the analysis [3, 4].

4. Study of High-Spin (EPR "Silent") Species: In high-spin systems with a substantial zero-field interaction studied at 9 or $35 \mathrm{GHz}$, often only a limited number of EPR transitions are within reach of the microwave quantum. For half-integer spin systems, this situation results in very complicated EPR spectra, which are spread over a large field range. For integer spin systems, these transitions may even be "spin forbidden", which makes the paramagnetic species "EPR silent". Higher and higher frequencies eventually will create a situation where the electron Zeeman interaction will dominate over the zerofield splitting. This will result in greatly simplified "first-order" EPR spectra in which the zero-field interaction can be treated as a perturbation that shows up as a splitting pattern. Therefore, in particular, high-spin transition-metal complexes are successfully studied using multi-high-frequency EPR.

5. Orientation Selection and Spectral Resolution in Double Resonance Experiments: Paramagnetic species, of which the EPR spectra, e.g., at X-band, are dominated by hyperfine interactions, are often difficult to study using electronnuclear double resonance (ENDOR). This is because the spectral features corresponding to the canonical orientations are not well resolved. This problem can be alleviated by increasing the microwave frequency and magnetic field. HF-EPR spectra usually are dominated by the anisotropic Zeeman interaction and facilitate orientation selection by adjusting the magnetic field at "canonical positions". Also, the ENDOR frequencies of the various nuclei will be separated better and better at higher and higher frequencies. Therefore, high- 
field ENDOR is a very powerful tool in the study of radical enzymes as well as metalloproteins.

Early high-field EPR spectrometers were usually built with one of these benefits in mind. The recent book of Möbius and Savitsky "High-field EPR applications on proteins and their model systems" [5] provides an excellent review on the evolution of high-field EPR instruments and various technical aspects. For completeness, we will reproduce the properties of early "first-generation" high-frequency EPR spectrometers from their review in the form of Table 1. The early projects to construct a high-frequency EPR spectrometer were mostly aimed at strong paramagnetic samples, such as radicals and concentrated paramagnetic crystals [6-12].

Lebedev et al. [13-16] were the first to systematically explore the application of HF-EPR for diluted paramagnetic systems such as spin labels. In 1977, they constructed the first high-sensitivity EPR spectrometer at $150 \mathrm{GHz}$ using a singlemode $\mathrm{TE}_{011}$ cylindrical resonator reaching an absolute sensitivity of $4 \times 10^{7}$ spins [17]. With this spectrometer and its successors, they extensively studied radicals and spin-labeled compounds with special interest in $g$-resolution and dynamics. Another milestone was reached in 1988 by Freed et al. [18], who built the first $250 \mathrm{GHz}$ continuous-wave (CW)-EPR instrument [18]. Again, spin label studies were the motivation to develop the instrument. Their "1-mm EPR" spectrometer employed oversized waveguides and polyethylene lenses to propagate the millimeter waves. In this sense, they built the first "quasi-optical" EPR spectrometer. CW and pulsed EPR as well as ENDOR at $95 \mathrm{GHz}$ (W-band) was realized for the first time by Schmidt et al. [19-21] and Möbius et al. [22-24]. In 1996, the Bruker company introduced the first commercial pulsed (and CW) EPR spectrometer at $95 \mathrm{GHz}[25$, 26]. Further developments in millimeter-wave (mm-wave) components at W-band allowed for improvements in excitation power and detection sensitivity [27]. The 95-GHz EPR spectrometer provides the optimum compromise between sample handling and absolute sensitivity. The technicians from the Donetsk PhysicalTechnical Institute (Ukraine), who were instrumental in the development of the mm-wave bridges $(95-150 \mathrm{GHz})$ of the Lebedev group, introduced pulsed $\mathrm{mm}$ wave bridges at 95 and $130 \mathrm{GHz}$ using high-power impact avalanche and transit time (IMPATT) amplifiers (200 $\mathrm{mW}$ at $95 \mathrm{GHz}$ and $50 \mathrm{~mW}$ at $130 \mathrm{GHz}$ ). These instruments were produced on a commercial basis and found their way to several laboratories in the world [28-33]. The increasing interest in higher-frequency EPR spectrometers $(>200 \mathrm{GHz})$ has stimulated the systematic application of quasioptical techniques in mm-wave propagation and resonator coupling and was initiated by Smith et al. [34] and Freed et al. [18, 35]. Currently, these groups are also exploring the benefits of high-power $(1 \mathrm{~kW}) \mathrm{W}$-band excitation. To control these high powers and maintain a very short system dead-time, advanced quasioptical techniques have proven to be essential [36, 37]. Also pulsed EPR at frequencies higher than $150 \mathrm{GHz}$ (using quasi-optical spectrometers) is being pursued in an increasing number of laboratories [38-42].

Although the most recent developments are focused on pulsed and time-resolved techniques, with high-sensitivity detection, the interest in wide-band HF-EPR to 


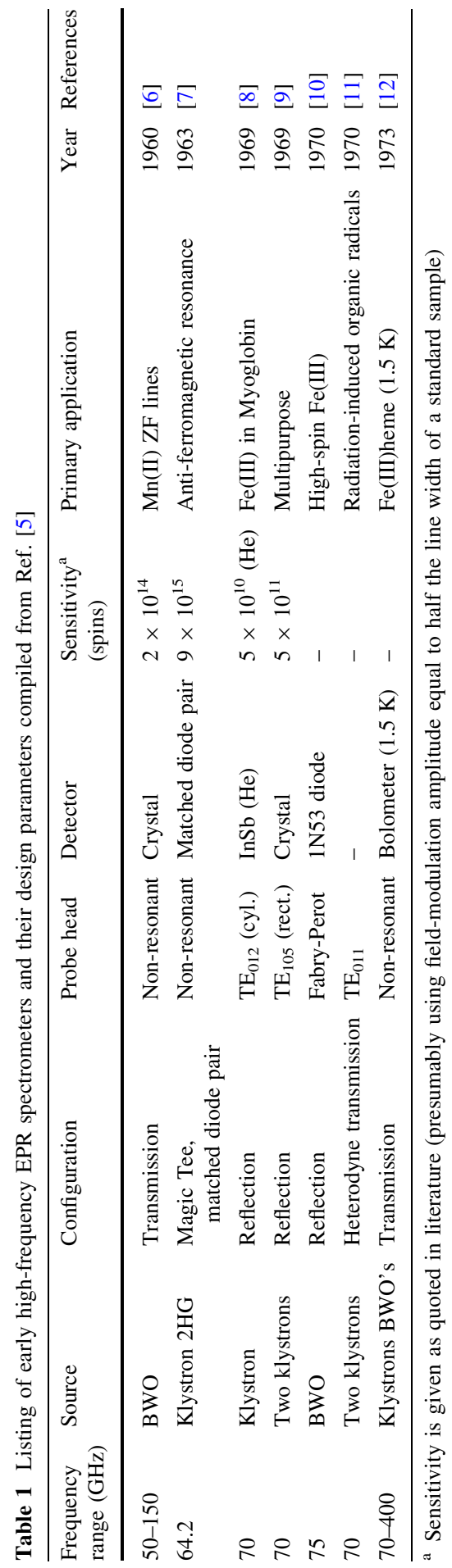


study strongly paramagnetic samples remains. Brunel et al. [43-45] realized a multifrequency HF-EPR spectrometer, which is optimized for the study of high-spin systems and radical species. Several laboratories followed similar schemes and developed multi-high-frequency instruments dedicated to a particular class of samples [46-49]. These setups do not need to be particularly sensitive and are less suited for work on diluted species and double resonance experiments. On the other hand, the microwave propagation system in these spectrometers can be kept relatively simple, which facilitates the implementation of multi-frequency operation and sample change. In this review, we will discuss the different components of a high-field, high-frequency EPR spectrometer and illustrate the various choices that can be made and their effect on the range of EPR applications that can be handled with a particular spectrometer.

\section{Sources}

The choice of microwave source is, of course, strongly related to the required power and frequency range. Also, the requirement of pulsed operation will be relevant to the choice of the microwave oscillator.

\subsection{Klystron and Gunn Oscillator}

In the last decade, the traditional microwave source in EPR, i.e., the klystron, has been phased out and replaced by the Gunn oscillator. The advantages of the klystron (very low-phase noise, high power, frequency control) have been offset by their poor availability and declining reliability. Recent developments in Gunn oscillator technology enabled the development of devices matching the performance of klystrons with the additional advantage of solid-state operation (low power consumption) and low replacement costs. Up to $150 \mathrm{GHz}$, both klystrons [22] and Gunn oscillators [22, 50, 51] can be employed in high-frequency CW-EPR spectrometers. For pulsed operation, however, they are less well suited. Gunn output powers are rather low (typically $50 \mathrm{~mW}$ at W-band) and high-frequency $\mathrm{CW}$ klystrons have a limited lifetime [23].

\subsection{Multiplied Sources}

In recent years, mm-wave and terahertz technology has been revolutionized by the use of varactor multipliers [52-55]. These devices are typically used in multiplier chains or "frequency extenders". At the input of these assemblies, a source in the "lower frequency range" for which power amplifiers are readily available (currently up to $95 \mathrm{GHz}$ ) is amplified and fed into, e.g., a chain of doublers. This way, in principle, frequencies can be generated in the $\mathrm{THz}$ range. For frequencies up to $600 \mathrm{GHz}$, appreciable powers can be reached (in the $\mathrm{mW}$ range). Usually, the low frequency input can be generated from very stable sources (including synthesizers, phase-locked Gunn or yttrium iron garnet (YIG)-based oscillators). Varactor multipliers can cover an extended frequency range (full waveguide band) and are 
therefore well suited to be incorporated in EPR sources as well as in test-andmeasurement sweep generators. A multiplication scheme has the additional advantage that, due to the non-linearity of these devices, the isolation of a pulsed excitation signal (i.e., the on/off ratio) is increased considerably ( $>50 \mathrm{~dB}$ ) [56]. The ease of operation and the possibility to integrate these devices in heterodyne as well as homodyne configurations lead to the expectation that these may be the sources of the future. Several groups already have integrated these designs in their HF-EPR spectrometers $[38,41,42,56]$.

\subsection{IMPATT Oscillator}

IMPATT oscillators have been developed and employed especially in the former Soviet Union. Although IMPATT diodes are primarily used in noise sources, they can be successfully used as phase stable oscillators when they are injection locked to a stable (low-power) signal. Lebedev et al. $[16,57]$ in Moscow can be regarded as the pioneers of high-frequency EPR. Their success was partly due to the use of an excellently designed microwave bridge based on an injection-locked IMPATT oscillator [16, 57] operating at $140 \mathrm{GHz}$. The IMPATT technology also has been successfully adapted to enable pulsed operation. The Donetsk Physical-Technical Institute (Ukraine) constructed several pulsed EPR bridges operating at 95 and $130 \mathrm{GHz}$ with output powers of 200 and $50 \mathrm{~mW}$, respectively. In combination with a single-mode resonator, these bridges enable excellent performance in pulsed EPR applications including pulsed ENDOR [28].

\subsection{Very High-Power Sources up to $140 \mathrm{GHz}$}

In some applications, very high microwave power is needed. Griffin et al. [50, 58] have been experimenting successfully using a gyrotron to irradiate electron spins at $130 \mathrm{GHz}$ in a dynamic nuclear polarization (DNP) experiment in combination with solid-state NMR. As an EPR source, however, gyrotrons are less suited because of their relatively high amplitude noise and problems in implementing pulsed operation. Extended interaction klystron (EIK) amplifiers at $95 \mathrm{GHz}$ have been used by Freed et al. (Cornell) [36] and Smith et al. (St. Andrews) [37, 59]. The EIK amplifier can deliver a peak power of $1000 \mathrm{~W}$ at $95 \mathrm{GHz}$ ! Theoretically, with this power one can imagine performing pulse experiments with $1 \mathrm{~ns} \pi / 2$ pulses that excite a complete EPR spectrum (of a spin label). This opens a whole new window of motion dynamics, as demonstrated by Freed et al. [3]. The microwave technology to handle these high powers requires a completely new approach to suppress unwanted reflections and damage to the detection system. This radical approach is followed by Smith et al. in the "HYPER Project" [37, 60].

\subsection{Far Infrared (FIR) Lasers}

FIR lasers occasionally have been used in early spectrometer designs. Their extended frequency coverage makes them well suited for multi-frequency CW-EPR studies of, e.g., high-spin systems $[43,44]$. The relatively high attainable power has 
been used to demonstrate the possibility of performing pulse EPR experiments at $604 \mathrm{GHz}$ [61-63]. Their poor amplitude stability, however, has prevented these sources form being employed widely.

\subsection{Backward Wave Oscillator (BWO)}

BWOs are used by several groups mainly focused on the study of molecular magnetism [46-49]. These oscillators combine a relatively high power with excellent frequency tuning possibilities. Also, their phase noise is favorable. Unfortunately, they are poorly available (mainly from sources in the former Soviet Union), require high-voltage power supplies and have to be operated in a local magnetic field, which can be easily disturbed by the stray field of the EPR magnet. Therefore, the use of these devices is rather limited.

\subsection{Orotron}

The orotron [64], also called "ledatron" [65], is a rather esoteric device, which functions somewhat like a free electron maser (gyrotron) but does not need a high magnetic field due to the use of a special grated electrode inside the vacuum tube $[66,67]$. This makes it possible to reach frequencies as high as $360 \mathrm{GHz}$. Only Möbius et al. [68] demonstrated the use of the orotron in EPR. It turns out that pulsed operation for this device is also possible [5]. Up to now, this device has not been shown to operate as an amplifier.

\subsection{Free Electron Laser}

The free electron laser is a very versatile mm-wave source because of its high power and large frequency range. In principle, it is also possible to realize pulse operation. However, this source is only available in large-scale infrastructures in which it is primarily used for other applications. The beam properties are usually not optimized for EPR experiments. Nevertheless, some groups are involved in adapting this instrument for EPR applications [69]. The future perspective for this project is to enable high-power pulse EPR experiments at very high frequencies (up to $4 \mathrm{THz}$ ).

\subsection{Phase Noise Considerations}

The most important parameters of a source are its frequency range, power, amplitude noise and phase noise. Of these, the phase noise has the strongest impact on the overall performance of the spectrometer, in particular when operating in $\mathrm{CW}$ mode. Although the requirements are somewhat more relaxed in pulsed experiments, phase noise can still affect, e.g., the spectral resolution in Fourier transform EPR. Therefore, great care has to be taken to minimize phase noise from the source or to minimize its effects, e.g., by equalizing path lengths in the mm-wave bridge (see below). Figure 1 shows the phase noise characteristics of commercially available sources scaled to $90 \mathrm{GHz}$. For this, the phase noise level is increased by $20 \log N$, where $N$ is the frequency multiplication factor. A free running Gunn 


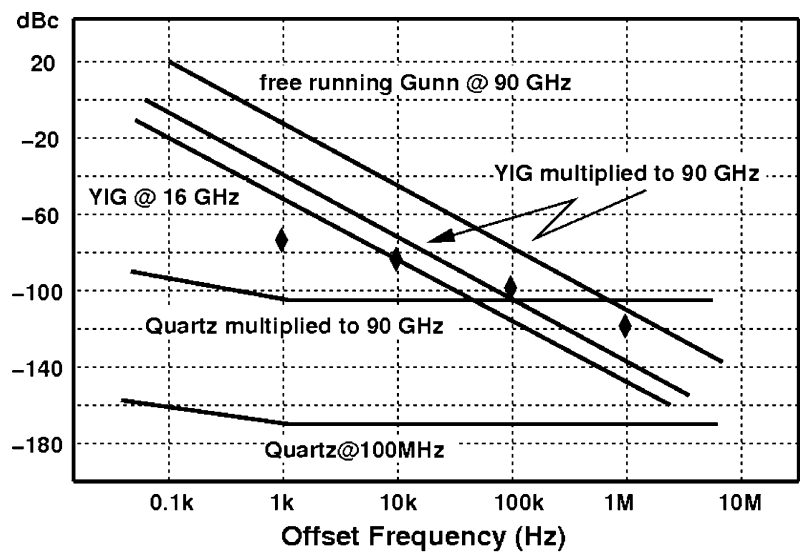

Fig. 1 Phase noise characteristics of commercially available sources scaled to $90-\mathrm{GHz}$ center frequency. Filled diamonds represent the phase noise of YIG-based synthesizers and DRO sources multiplied to $90 \mathrm{GHz}$ (G.M. Smith, pers. commun.)

oscillator has a $30 \mathrm{~dB}$ per decade frequency dependence. A YIG oscillator multiplied to $90 \mathrm{GHz}$ has an overall $25 \mathrm{~dB}$ better performance. Usually, the performance of these oscillators can be improved by locking to a crystal. Figure 1 also shows the characteristic of a 100-MHz crystal multiplied to $90 \mathrm{GHz}$. Indeed, a phase-locked Gunn oscillator shows a phase noise around $-90 \mathrm{dBc}$ at $100 \mathrm{kHz}$ and -80 at $10 \mathrm{kHz}$, which is a considerable improvement over the free running configuration. It would, however, seem more convenient to use multiplied YIGbased sources since these provide a wider frequency range. As shown in Fig. 1, these devices show an improved phase noise with respect to the free running (YIG) case up to $100 \mathrm{kHz}$. Above $100 \mathrm{kHz}$, the trend of the free running oscillator takes over, and locking no longer seems to be an advantage. For CW-EPR, however, the range from 1 to $100 \mathrm{kHz}$ is most important. Commercially available dielectric resonator oscillator (DRO) sources show a similar phase noise characteristic. They are cheaper than YIG-based synthesizers, but are limited to a fixed frequency. Therefore, they are mostly suited as local oscillators in a heterodyne configuration. Both stabilized YIG and DRO oscillators are considered the most appropriate sources for multiplication to mm-wave frequencies [70].

\section{Detection Systems}

\subsection{Schottky Diodes}

In conventional CW-EPR spectrometers at X- and Q-band, Schottky diodes are the standard detectors [1]. At higher frequencies, their performance, however, drastically declines mainly because of increased $1 / f$ noise characteristics. Therefore, they are rarely used as signal detectors in HF-EPR spectrometers, but more as diagnostic tools and power meters [34]. However, in applications where a high 
dynamic range is of importance, Schottky detectors are preferred even over InSb bolometers.

\subsection{InSb Bolometer}

In CW HF-EPR systems, helium-cooled InSb bolometers have been extensively used because of their unrivaled sensitivity and reasonable dynamic range. Since the typical bandwidth in which a bolometer can be used is limited to a few $\mathrm{MHz}$, they are less suited for pulsed detection. Optimal performance is reached when the sensor is positioned in a single-mode waveguide matched to a feed horn. They are typically used in a homodyne configuration, i.e., a small local oscillator signal of a few microwatts at the excitation frequency is applied to provide the proper bias to the detector. Due to the waveguide configuration, the operation is limited to the waveguide bandwidth. Untuned $\mathrm{InSb}$ mixers can reach a noise temperature of $2000 \mathrm{~K}$ at $90 \mathrm{GHz}$ [34]. High-performance CW HF-EPR spectrometers have been described making use of multiple frequencies and multiple He-cooled InSb mixers mounted in a single cryostat $[34,71]$. Bolometers can also be used in a broadband configuration when they are mounted in an integrating cavity. These instruments usually make use of a sweepable source such as a BWO. Although the sensitivity is reduced, these systems are very well suited for multi-frequency measurements on magnetic materials [43-45, 72, 73]. Moreover, they are relatively easy to construct because the microwave propagation system as well as the sample probe head can be kept very simple.

\subsection{Schottky Mixers}

In pulsed EPR spectrometers, Schottky mixers are exclusively used because of their large instantaneous bandwidth. Recent technological developments have pushed the accessible frequency range to the $\mathrm{THz}$ domain. Due to their conversion loss (typically around $8 \mathrm{~dB}$ ), Schottky mixers are usually combined with a low noise amplifier (LNA) in the "radio-frequency" (RF) port. Up to $95 \mathrm{GHz} \mathrm{LNAs}$ are currently available $[51,74]$. It should be noted that an LNA increases the sensitivity to small signals, but, on the other hand, reduces the effective dynamic range of the mixer. For higher frequencies, often a heterodyne detection configuration is employed. Here, the mixer serves to convert the detected RF signal down to an intermediate frequency (IF, which is the difference between the RF and local oscillator [LO] frequency) below $18 \mathrm{GHz}$. This IF signal is then amplified using an LNA. In principle, fundamental mixers (i.e., mixers where RF and LO are in the same frequency band) are preferable because of their low conversion loss. At higher frequencies $(>150 \mathrm{GHz})$, however, they are only available as "single-ended" mixers, i.e., they require an external diplexer to combine RF and LO since these will share the same port. Consequently, the port isolation and amplitude noise suppression characteristic for double balanced mixers are lost in the single-ended configuration. Table 2 lists the noise characteristics of typical bolometer and Schottky mixers. Here, it should be noted that the figures of merit, noise equivalent power (NEP) and the noise temperature of a mixer, are not directly comparable in 
Table 2 Properties of detectors used in high-frequency EPR

\begin{tabular}{lllcc}
\hline Detectors & Frequency range & $\mathrm{NEP}^{\mathrm{a}}\left(\mathrm{W} / \mathrm{Hz}^{-1 / 2}\right)$ & $T_{\text {mix }}{ }^{\mathrm{b}}(\mathrm{K})$ & IF band \\
\hline Astronomical bolometer & $\mathrm{THz}$ & $10^{-18}$ & & $1 \mathrm{kHz}$ \\
Bolometer mixer & $100 \mathrm{GHz}$ & $10^{-13}$ & 2000 & $1 \mathrm{MHz}$ \\
Schottky detector & $150 \mathrm{GHz}$ & $10^{-12}$ & & $1 \mathrm{GHz}$ \\
Zero bias & $800 \mathrm{GHz}$ & $10^{-11}$ & 400 & $>10 \mathrm{GHz}$ \\
Schottky mixer & $100 \mathrm{GHz}$ & & 500 & $>10 \mathrm{GHz}$ \\
& $200 \mathrm{GHz}$ & & 1000 & $>10 \mathrm{GHz}$ \\
\hline
\end{tabular}

${ }^{\mathrm{a}} N E P$, noise equivalent power, ${ }^{\mathrm{b}} T_{\text {mix }}(\mathrm{K})$, noise temperature (double side band) with matched IF source

the EPR context. The NEP of a bolometer is a sensitivity measure relevant to wideband incoherent radiation (e.g., in astronomy), whereas the noise figure of a mixer relates to narrow-band coherent detection (as relevant in EPR). Undoubtedly, the absolute sensitivity of the astronomical bolometer is unrivaled, but its strongly limited dynamic range makes it unsuitable for EPR applications.

\subsection{Millimeter-wave Vector Network Analyzer (MVNA)}

The MVNA developed by the company ABmm has an unrivaled dynamic range and has been used in several laboratories involved in multi-high-frequency EPR [45, 49, 57, 73]. The sources up to $300 \mathrm{GHz}$ are based on YIG oscillators in combination with harmonic generators. Alternatively, up to $900 \mathrm{GHz}$, multiplied phase-locked wide-band Gunn sources can be used. The detection system consists of harmonic mixers (pumped by a YIG oscillator or a second Gunn source). The MVNA is particularly suited as a diagnostic tool to characterize resonators and quasi-optical systems.

\section{Probe Heads}

The probe head or sample holder obviously is one of the key components of an EPR spectrometer. A wide range of models has been described as part of a high-field, high-frequency setup. The variety in designs is mainly driven by the particular types of samples (liquid, solids, crystals) and the additional experimental requirements (light access, crystal rotation, RF). The developed devices range from a simple nonresonant transmission "cuvette" to a single-mode resonator with crystal rotation, ENDOR coil and optical access. The general "figure of merit" of a sample holder is given as $Q \eta$, where $\eta$ represents the filling factor and $Q$ is the quality factor of the probe head. Typical values range from ten to a few hundred. $Q \eta$ enters in Eq. (2) (Sect. 7) which describes the general sensitivity of a spectrometer. In the following, we will describe the most widely used probe heads and their applications in some detail. 


\subsection{Fabry-Perot (FP) Resonators}

A wide variety of FP resonators have been developed for various applications. The open structure of the FP resonator makes it particularly useful for applications involving optical access as well as ENDOR. These benefits have been extensively demonstrated by Möbius et al. [22, 24], who applied a bi-confocal FP resonator at W-band. Also, Schmidt et al. $[19,75]$ selected an FP resonator for their first W-band experiments. Freed et al. [18, 35, 76] described semi-and bi-confocal FP resonators operating at $250 \mathrm{GHz}$. These resonators were coupled through an iris. Smith et al. [34] demonstrated that quasi-optical coupling through a mesh provides the benefits of induction-mode detection, which greatly enhances the decoupling of the excitation and detection beams. This detection scheme is illustrated in Fig. 2. The corrugated waveguide allows the HE11 mode, which is equivalent to a free space fundamental Gaussian beam, to propagate to the probe head, while its polarization is conserved. In essence, one can consider the linear polarized beam as composed of two counteracting circular polarized beams. On magnetic resonance, however, the energy of only one circularly polarized beam is absorbed. Therefore, the reflected beam will contain a slight excess of cross-polar component, which contains the EPR signal. The EPR signal can be separated off from the reflected beam by a grid polarizer. This very elegant scheme can be utilized with all probe heads of cylindrical symmetry around the axis of the guide and with matching (in-plane) $\mathbf{B}_{1^{-}}$ orientation. Apart from the Fabry-Perot resonator, this scheme has been demonstrated for non-resonant probe heads, as well as for a $\mathrm{TM}_{110}$ resonator [60].

Although mesh coupling in the Fabry-Perot resonator, in principle, does not allow for variable coupling, in practice this does not seem to be a problem. The mesh can be chosen such that a slight overcoupling is achieved on an empty

Fig. 2 Principle of inductionmode detection: the resonator is excited by a linear polarized beam that can be considered as two counter-rotating circular polarized beams. On EPR absorption one of these beams is attenuated, which results in a cross-polar contribution in the reflected beam. (Figure reproduced from Ref. [34] with permission from American Institute of Physics.)

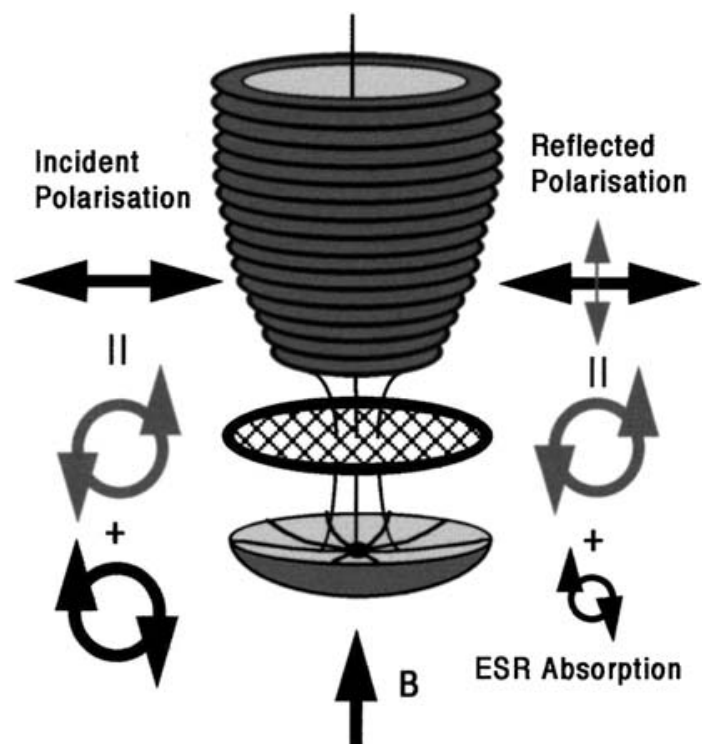



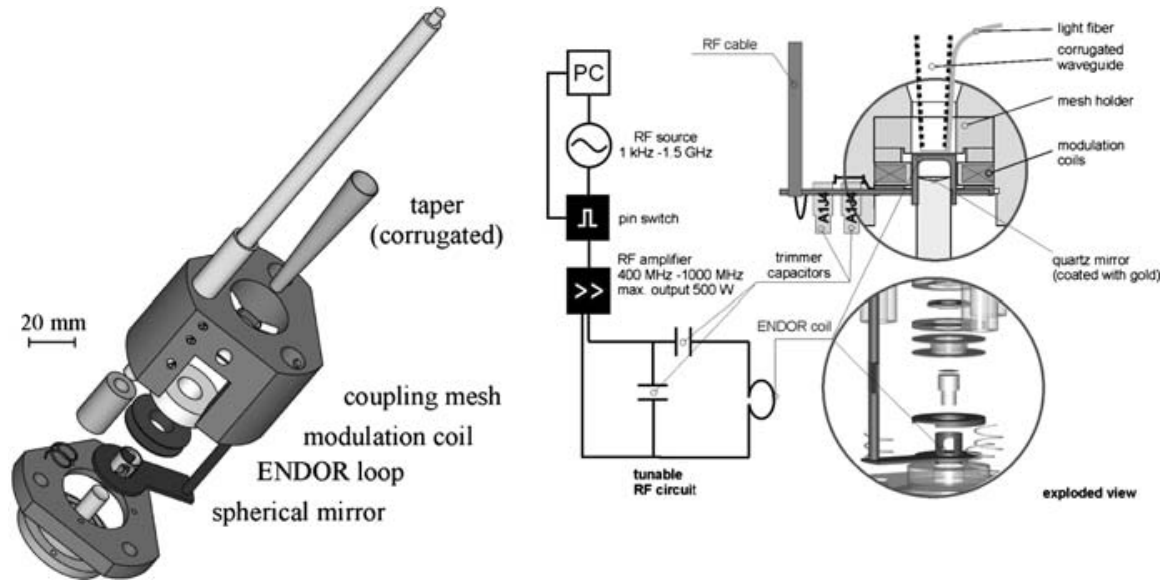

Fig. 3 Magnified view of Fabry-Perot resonator with single-loop ENDOR coil at $360 \mathrm{GHz}$ indicating the different components. The RF loop generates a $B_{2}$-field perpendicular to the mm-wave $B_{1}$-field and the (vertical) $B_{0}$-field. The sample is placed on the gold-coated spherical mirror. For ENDOR experiments, the coupling mesh is removed (creating a non-resonant probe head). For details see Refs. [79, 80]

resonator. By varying the amount of sample, the coupling can be adjusted to critical levels. Attempts to achieve variable coupling by using a double mesh also have been reported [35]. The FP resonator is the best resonator to accommodate aqueous liquid samples. Several arrangements have been demonstrated [77, 78]. Möbius et al. [79, 80] presented a Fabry-Perot resonator operating at $360 \mathrm{GHz}$ that allowed (CW) ENDOR experiments. This configuration is depicted in Fig. 3.

\subsection{Non-Resonant Sample Holders}

The simplest sample holders used in high-field EPR consist of just a plastic or quartz cup inside a smooth, oversized waveguide. This configuration is very simple and effective when employed in a multi-frequency, high-field EPR setup optimized for the study of strong paramagnetic centers such as molecular magnets. The instrument typically consists of a multi-frequency source (e.g., FIR laser, BWO), an oversized waveguide system including the sample holder, and a large bandwidth detector, e.g., a bolometer (see below). In the simplest ("optical") configuration, the sample holder is positioned in "transmission". In the original "Grenoble configuration" $[45,72]$, a double copper pipe is inserted into the sample cryostat. Mirrors inserted into the pipes allow for $90^{\circ}$ bends connecting the source and detector pipes as well as the transmission sample holder. The limitations in sensitivity for this arrangement are mainly caused by the standing waves, which will occur in the smooth waveguides on both sides of the sample holder. The standing waves are caused by mode conversion effects in the smooth guides and will convert the source phase noise into amplitude noise at the detector. Due to incomplete control of the $\mathrm{mm}$ wave phase, often a mixture of absorption and dispersion is recorded. 
The use of quasi-optics and corrugated waveguides in combination with induction-mode detection, as described above, will greatly increase the achievable sensitivity. It has been demonstrated that non-resonant probe heads using tapered (corrugated) waveguides can achieve similar concentration sensitivity as a singlemode resonator $[42,60,81]$. The decoupling of the cross-polar components to nonresonant probe heads can be further improved by using an adjustable roof mirror underneath the sample holder, correcting phase and polarization mismatches caused by the sample itself. The diameter of the non-resonant sample holder can be reduced to quasi-single-mode size allowing for reduced sample volumes and the mounting of ENDOR coils as well as optical access [73, 81].

\subsection{Single-Mode Resonators $\left(\mathrm{TE}_{011}\right)$}

At high frequencies, the single-mode $\mathrm{TE}_{011}$ cylindrical resonator is considered to be the optimum solution to achieve maximum absolute sensitivity. Obviously, the dimensions decrease as the frequency increases, and its mechanical construction becomes proportionally challenging. Currently, the record is set at $275 \mathrm{GHz}$, as demonstrated by Schmidt et al. [39, 82]. The single-mode resonator has the highest possible power conversion ratio. Already with moderate power, a substantial $B_{1}$ field can be generated. Therefore, pulsed EPR applications preferably make use of a single-mode resonator. At $95 \mathrm{GHz}$, an incident power of approximately $100 \mathrm{~mW}$ is necessary for a $\pi / 2$ pulse of $50 \mathrm{~ns}$ (cavity $Q=2000$ ) [28]. At $180 \mathrm{GHz}$, this is $20 \mathrm{~mW}(Q=1500)$ [38]. For $360 \mathrm{GHz}$, only $4 \mathrm{~mW}$ would be needed for a $50 \mathrm{~ns} \pi / 2$ pulse $(Q=1500)$ [39]. Several coupling schemes are described. The iris in the cylinder wall can be adjusted using a metal tip on a dielectric slab [22]; alternatively, the iris can be placed in the plunger wall and the coupling can be adjusted using a sliding short [83]. A convenient way to adjust the coupling is by rotating the resonator around the waveguide coupling to the iris hole in the cylinder wall. This mode allows a large iris hole, which facilitates the scaling up to higher frequencies $[20,28,82]$. A specific challenge is to combine the resonator with an ENDOR coil enabling pulse ENDOR applications. An obvious choice is to mount the coils in a pseudo-Helmholtz configuration. To allow the RF field to penetrate the resonator, slits are machined in the resonator body. These slits also can serve as optical access $[20,82]$. At lower frequencies $(90-150 \mathrm{GHz})$, it has been demonstrated that the resonator cylinder itself can be formed from a "ribbon coil" [58]. It should be noted also that at W-band frequencies a split pair magnet can be used, so that the $\mathrm{TE}_{011}$ resonator can be oriented vertically, thus enabling top loading of the sample $[25,26]$.

\subsection{Other Resonator Designs}

At lower frequencies $(<35 \mathrm{GHz})$, dielectric and loop gap resonators have become very popular as probe head for pulsed applications. These designs are, however, difficult to scale to higher frequencies because of the microscopic dimensions that are required. For W-band (94 GHz), Hyde et al. [84-86] constructed a five-loop four-gap resonator using electrical discharge machining. The sample access in this resonator $(0.6 \mathrm{~mm})$ is comparable to that of a cylindrical $\mathrm{TE}_{011}$ resonator at $\mathrm{W}$-band. Some 
attempts have been made to construct alternative resonators exhibiting extremely high $Q$-values, such as the whispering-gallery-mode (WGM) resonators [87-90]. Sample handling in these resonators is, however, problematic and up to now the WGM resonator has not been employed regularly in high-field EPR experiments. A new "non-radiative" design from the same group, however, seems to be promising. In particular, the coupling and open structure of the cavity are advantageous for optical access, ENDOR applications as well as crystal rotation [91-94].

\section{Magnet Technology}

The commonly employed field sweep in the EPR experiment sets out specific requirements on the magnet technology used in high-field, high-frequency spectrometers. Magnetic fields up to $18 \mathrm{~T}$ are usually generated using cryogenic magnets. These should be specifically designed to allow large field sweeps. Over the whole field range, the homogeneity should be good (typically $10^{-4} \mathrm{~cm}^{-3}$ sphere). At specific field values (e.g., around $g=2$ ) additional homogeneity can be reached using shim coils (up to $10^{-6} \mathrm{~cm}^{-3}$ ). For accurate field sweeps in a small range $( \pm 1500 \mathrm{G})$, cryogenic sweep coils can be used. When using liquid helium-cooled cryogenic magnets, one should realize that during a large field sweep (using the main coil), substantial helium boil-off will occur. Since NMR magnets are designed to be stabilized at a fixed field, the leads are demountable. For EPR magnets, one usually chooses a design with fixed leads. Although this leads to a somewhat higher static boil-off, the operation of the magnet is greatly simplified. Recently, cryogenfree magnet technology has been introduced in the field of EPR [95]. The great advantage of these systems is the ability to continuously sweep the main coil without the associated helium costs. In addition, the system can be completely switched off over periods when it is not in operation. The magnet can be cooled down typically within $50 \mathrm{~h}$ (e.g., a weekend) by simply turning on a switch.

While selecting a cryomagnet for high-field EPR, the following options have to be considered:

1. Liquid Helium or Cryogen free operation? The advantages of cryo-free operation are the reduced helium costs in general, the ability to continuously sweep the main coil, and a "graceful recovery" after a quench. The drawbacks are the potential vibration contribution to the signal to noise ratio of the spectrometer, and the acoustic load in the laboratory.

2. Fixed or demountable leads. The advantages of fixed leads are the ease of operation, and reduced helium costs when the main coil is operated regularly. The drawback is the steady-state slightly increased helium boil-off.

3. Sweep coil or not? A separate sweep coil enables high resolution sweeps over a small field range (typically the $\mathrm{g} \approx 2$ range). However, due the inductive coupling between sweep and main coil, the sweeps of both coils are non-linear. This effect does not occur when only one coil is present in the magnet.

4. Thermo switch or not? With a thermo switch installed, the main coil sweeps will show a hysteresis depending on the sweep rate. 
5. Warm bore or cold bore? A warm bore increases the size of the magnet (and its price) for the same field. On the other hand, the user is completely free to install any type of sample cryostat.

For fields higher than $18 \mathrm{~T}$, one can make use of large-scale facilities providing resistive magnets of the "Bitter" type. With power supplies of $20 \mathrm{MW}$, fields of $32 \mathrm{~T}$ are routinely reached $[57,73]$. In the standard configuration, these systems are limited in field stability and homogeneity, but they are very well suited for the study of strong paramagnetic centers such as molecular magnets. With some additional effort, the field homogeneity can be substantially improved either by a special coil design or by using ferro-shims [96].

While standard EPR systems typically employ a Hall stabilization scheme of the magnet, this design has to be abandoned in a cryomagnet. Due to the high inductance of the coil, electronic stabilization is not possible. In many cases, the bore of the magnet is completely occupied by the sample cryostat, leaving no room for a field sensor. Usually, the magnetic field axis has to be calibrated using a standard paramagnetic sample, e.g., $\mathrm{Mn}$ (II) in $\mathrm{MgO}$ [22]. The use of a sweep coil and a thermo switch unfortunately lead to non-linearity and hysteresis of the magnetic field sweep. By minimizing the inductive contact between the main and sweep coils, some components of the non-linearity can be suppressed. The field hysteresis can be minimized by letting the magnet power supply use "current jumps" at the start and end of a sweep [25, 26]. In the Bruker W-band system, the magnetic field is calibrated for a multiple of sweep rates using an NMR gaussmeter in the free bore of the magnet. A more direct solution to the calibration problem is to leave room in the magnet bore for a field sensor. In this way, the field axis can be recorded in parallel to the EPR amplitude.

\section{Spectrometer Configurations}

In the following, we will briefly discuss the most popular spectrometer schemes used in high-sensitivity instruments.

\subsection{Homodyne Bridges}

The mm-wave bridges up to $130 \mathrm{GHz}$ from Lebedev group are scaled copies of a standard homodyne X-band bridge (see Fig. 4a), in which the detector is replaced by a helium-cooled InSb element $[16,17]$. The InSb wafer is mounted inside a waveguide, which is immersed in a helium vessel. As sources, various low-phase noise oscillators have been used, i.e., BWO and klystron. More convenient are solidstate sources, such as Gunn oscillators and IMPATT sources. The reference arm allows full control of absorption and dispersion detection [57]. This homodyne bridge was also modified to perform pulse experiments [97]. The 95-GHz bridge developed by the group in Urbana $[51,83]$ is also of the homodyne type, but uses a standard Schottky diode detector. Especially in combination with a low-noise amplifier [51], the sensitivity is compatible with that of the bolometer bridges, i.e., 


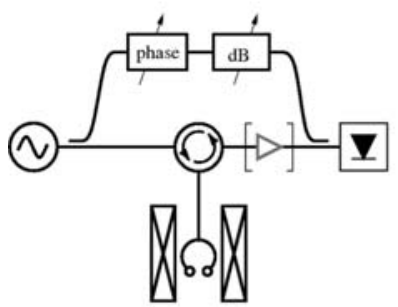

(a)

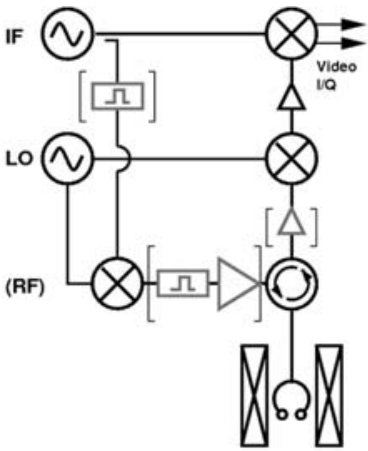

(b)

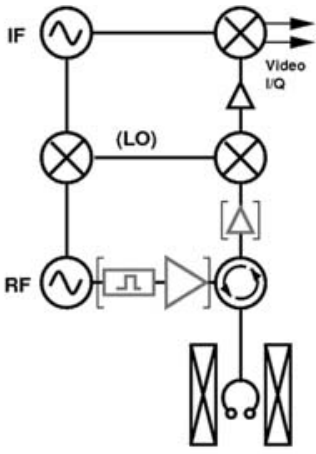

(c)

Fig. 4 Detection schemes in EPR. Optional elements are indicated between brackets. a Homodyne detection scheme. Optionally, a preamplifier can be used to compensate for the unfavorable sensitivity of a Schottky diode detector at high frequency. b Heterodyne detection scheme where pulse forming (optionally) can be achieved at the IF frequency. c Heterodyne detection scheme where pulses are generated at the RF frequency and LO is generated from RF and IF

the absolute sensitivity approached the theoretical limit of $10^{8}$ spins. The availability of a low-noise amplifier also facilitated the development of a homodyne pulsed W-band bridge by Goldfarb et al. [74].

The homodyne scheme can also be employed at much higher frequencies. Due to the increasing losses in single-mode waveguides, one is forced to switch to oversized propagation systems. It has been shown that smooth circular guides, although very convenient to use, have serious drawbacks concerning poor mode conversion, which leads to standing waves. This makes it difficult to control the local oscillator level at the detector and renders the system very sensitive to vibrations. With the systematic introduction of quasi-optics for high-frequency EPR, Smith et al. [34] have solved these typical problems for the most part. Figure 5 shows the functional layout of their setup. The spectrometer can work at 80$100 \mathrm{GHz}$ as well as $160-200 \mathrm{GHz}$ (using a doubled source). The power from the source is led through the quasi-optical propagation system, where a beam splitter couples out a portion of the power to feed the reference arm. The main part of the power is directed through a Faraday rotator to the resonator insert located in the variable-temperature insert of the magnet. In the induction-mode configuration, the probe head (either a Fabry-Perot resonator or a non-resonant sample holder) reflects the EPR signal in cross-polar polarization. This part is stripped off by a polarizer and is led to the detection system, which consists of a bolometer mixer. The local oscillator level and phase can be adjusted by using a movable roof mirror that reflects the power from the beam splitter through a polarizer to a subsequent polarizer, which combines the LO with the RF (EPR) signal. The combined LO and $\mathrm{RF}$ signal is directed to the detection diodes. In the depicted configuration, the detection diodes act as a balanced pair suppressing the source AM noise of the Gunn oscillator. In practice, the source noise is already very low and the setup is configured to use a single helium-cooled InSb bolometer mixer. The polarizer that reflects the EPR signal will transmit the directly reflected signal to a Schotty diode 


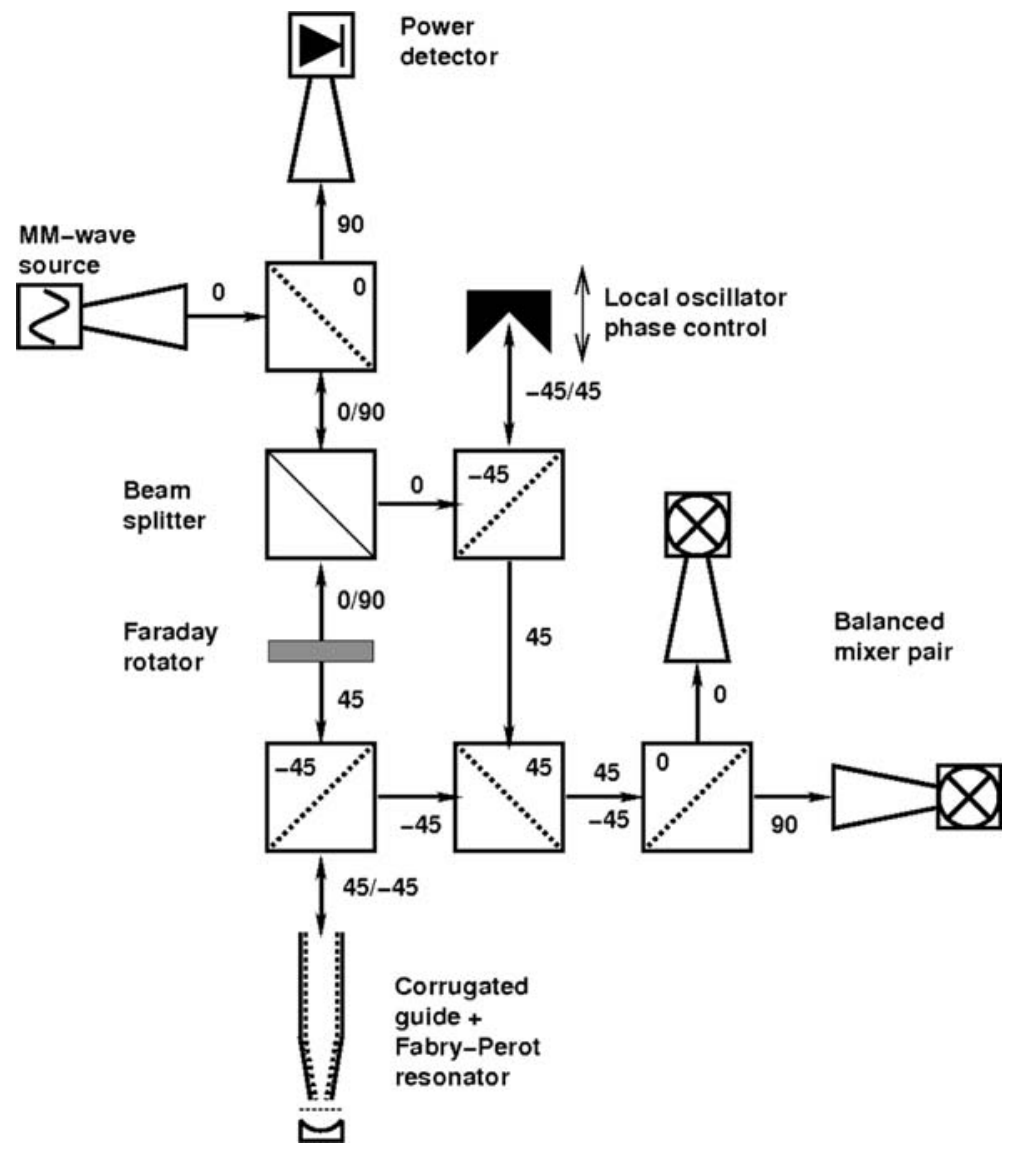

Fig. 5 Functional block diagram of a quasi-optical millimeter-wave homodyne HF-EPR bridge as introduced by Smith et al. [34]. For clarity, the focusing elements have been omitted. The polarization of the millimeter-waves is indicated as the angle of the $\mathbf{E}$-field vector with the normal to the propagation plane. For polarizers, the angle of the wires with the normal to the plane is indicated

detector, which can be used to tune the resonator. The spectrometer can be easily adapted to operate at different frequencies because the quasi-optical elements are usually broadband. In particular, when using the so-called unity-gain optics, only the matching layers of the Faraday rotator as well as the feed horns of source and detector have to be exchanged. Several groups have now used this design and constructed their own version of a homodyne quasi-optical CW-EPR spectrometer [71, 98, 99].

\subsection{Heterodyne Bridges}

For larger detection bandwidths, as is necessary in high-frequency pulsed and transient EPR applications, often a heterodyne detection scheme is adopted, in 
particular, when a low-noise amplifier for the frequency of interest does not exist or specifies a worse noise figure than the conversion loss of the fundamental detection mixer. In principle, one can choose from two basic heterodyne schemes (Fig. 4b, c). In both cases, one has to consider how to correlate the phase noise of the two sources. Schmidt et al. [19, 20] in Leiden, who pioneered the development of W-band pulsed EPR, used scheme in Fig. $4 \mathrm{~b}$ for the design of their bridge. The advantage of this scheme is that pulse forming can be performed in the IF channel at low frequency using affordable components. The drawback of this configuration is that the amplitude and phase noise of both the IF and LO sources is present in the RF channel, which could affect the CW-EPR performance. Möbius et al. [5, 23] in Berlin, therefore, opted for scheme in Fig. 4c for their W-band spectrometer. By balancing the paths lengths associated with the IF source, the video contribution of the phase noise of this DRO $(-80 \mathrm{dBc}$ at $10 \mathrm{kHz})$ could be suppressed by more than $46 \mathrm{~dB}$. Steinhoff et al. [100] used the same design in their 95-GHz instrument. Prisner et al. [38] used this principle in their $180-\mathrm{GHz}$ pulse EPR spectrometer. The $\mathrm{W}$ - and D-band bridges developed by Vladimir Krymov (Donetsk Physical-Technical Institute, Ukraine), however, used scheme in Fig. 4b, in which the pulses were still formed in the RF channel. In this case, LO and RF signals are derived from low (fixed) frequency stable oscillators and are multiplied using IMPATT multipliers ( $\times 13)$ [28]. The Bruker company in their W-band spectrometer also prefer the scheme in Fig. 4b, since it allows the pulse forming and detection to be performed at the IF frequency of $9 \mathrm{GHz}$ for which the pulsed X-band bridge can be used. In this way, a W-band system can be obtained as an upgrade to an X-band spectrometer [25, 26]. In practice, the difference between designs in Fig. $4 \mathrm{~b}$ and c will be minimal, when low-phase noise sources are employed, and LO and RF noise is suppressed by proper balancing of the optical path length of the branches in the mm-wave bridge.

At higher frequencies $(>150 \mathrm{GHz})$, quasi-optical bridges are used, and the fundamental Schottky mixers are only available in a single-ended configuration. This means that both LO and RF signals must enter through the same port. This requires diplexing the $\mathrm{LO}$ and RF signals in the quasi-optical bridge. This concept will complicate the layout of the bridge considerably, since both RF and LO signals need separate quasi-optical paths. This principle was applied in the pulsed EPR bridge at $275 \mathrm{GHz}$ by Schmidt et al. [39, 82]. Similar schemes were employed in the multi-frequency bridges operating at 120 and $240 \mathrm{GHz}$ developed by van Tol et al. [41, 81] and Reijerse et al. [42]. The functional block diagram of these systems is illustrated in Fig. 6. In CW mode, usually induction-mode detection is used. In this case, the signal is reflected from the polarizer and directed to the signal mixer. The directly reflected signal from the probe head is passed through the polarizer and fed through the Faraday rotator and subsequently reflected to the reference mixer. This mixer signal is used as the reference for the IF mixer. This has the advantage that the phase noise of the LO and RF sources is optimally correlated, since the optical path length of the signal and the reference is equal. In addition, phase drifts due to temperature variations in the probe head insert are compensated. The quasi-optical heterodyne spectrometers developed by van Tol et al. [41, 81] and Reijerse et al. [42] are based on unity-gain optics. This enables a multi-frequency operation since the optics are frequency independent. For a frequency change apart from the probe 


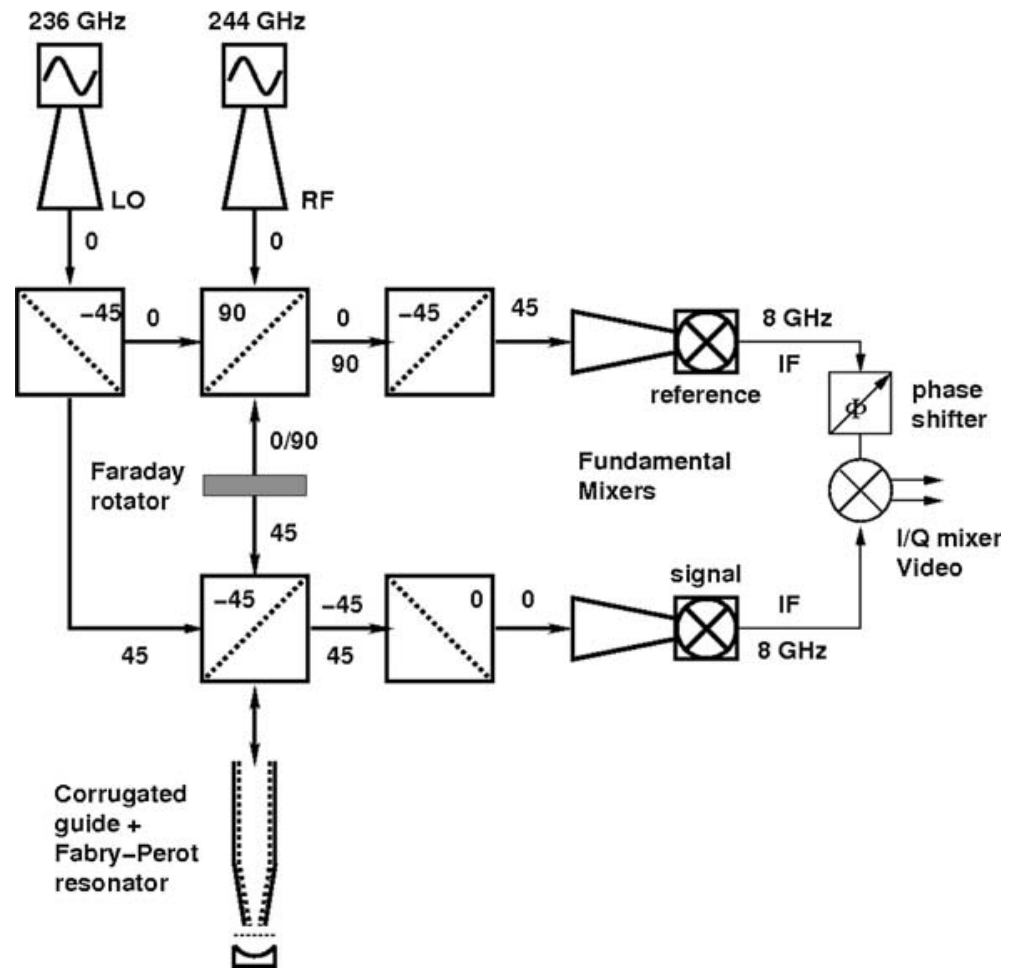

Fig. 6 Functional block diagram of a quasi-optical CW/pulse/transient mm-wave heterodyne HF-EPR bridge like the one describe by van Tol et al. [41, 81] and Reijerse et al. [42]. For clarity, the focusing elements have been omitted. The polarization of the mm-waves is indicated as the angle of the E-field vector with the normal to the propagation plane. For polarizers, the angle of the wires with the normal to the plane is indicated

head, only sources have to be modified (exchange of multipliers and feed horns), the mixers have to be exchanged, as well as the Faraday rotators. For very highfrequency (pulsed) EPR, these types of quasi-optical systems offer the highest flexibility in configuration concerning sources, detectors and probe heads.

\section{Sensitivity Considerations}

The fundamentals of spectrometer sensitivity in relation to the cavity and detector properties have been discussed in great detail by Feher [101] and Poole [1, 2]. The frequency dependence of the signal-to-noise from the $\mathrm{MHz}$ range up to $250 \mathrm{GHz}$ has been analyzed by Rinard et al. [2, 102-104]. Here, we will only treat the most relevant parameters related to source and detector noise. The general expressions for the spectrometer sensitivity have been derived by Poole [1] and are presented in several text books [105]. It relates the basic properties of the cavity and the noise floor of the detection system to the detectable number of spins. For the absolute sensitivity, one obtains: 


$$
N_{\min }=\frac{6 V_{\mathrm{C}} k T \Delta B}{\mu_{0} g^{2} \mu_{\mathrm{B}}^{2} S(S+1) B_{\mathrm{r}} Q_{\mathrm{u}}}\left(\frac{F k T \Delta f}{P_{\mathrm{W}}}\right)^{1 / 2} .
$$

By introducing the filling factor (approximated as $\eta=V_{\text {Sample }} / V_{\text {Cavity }}$ ), the concentration sensitivity can be written as:

$$
\frac{N_{\min }}{V_{\mathrm{S}}}=\frac{6 k T \Delta B}{\mu_{0} g^{2} \mu_{\mathrm{B}}^{2} S(S+1) B_{\mathrm{r}} Q_{\mathrm{u}} \eta}\left(\frac{F k T \Delta f}{P_{\mathrm{W}}}\right)^{1 / 2} .
$$

The following descriptions and values are used for the various parameters appearing in Eqs. (1) and (2): $V_{\mathrm{C}}$ is the volume $\left(\mathrm{m}^{3}\right)$ of the resonator, with the typical values $\mathrm{TE}_{102} @ 9.5 \mathrm{GHz}=1.1 \times 10^{-5} \mathrm{~m}^{3}$ and $\mathrm{TE}_{011} @ 90 \mathrm{GHz}=2 \times 10^{-8} \mathrm{~m}^{3}$; $k$ (Boltzman constant $)=1.38062 \times 10^{-23} \mathrm{~J} \mathrm{~K}^{-1} ; T$ (sample temperature) is typically room temperature $=300 \mathrm{~K} ; \Delta B$ is the line width of absorption signal $=0.1 \mathrm{mT}$; $B_{\mathrm{r}}$ is the resonant field (X-band, $340 \mathrm{mT}$; W-band, $3.4 \mathrm{~T}$ ); $Q_{\mathrm{u}}$ is the unloaded quality factor of the resonator (X-band, 5000; W-band, 2000); $\Delta f$ is the detection bandwidth $=1 \mathrm{~Hz} ; P_{\mathrm{w}}$ is the incident power on the resonator (X-band, $100 \mathrm{~mW}$; $\mathrm{W}$-band, $10 \mathrm{~mW}$ ); $F$ is the noise factor of the spectrometer (X-band diode, 100; $\mathrm{W}$-band bolometer, 20$)^{1} ; \mu_{0}$ is the magnetic susceptibility of vacuum $=4 \pi \times 10^{-7}$ $\mathrm{V} \mathrm{s} / \mathrm{Am} ; g$ is the $g$-factor $=2 ; \mu_{\mathrm{B}}$ is the Bohr magneton $=9.2838 \times 10^{24} \mathrm{~J} \mathrm{~m}^{2} / \mathrm{V} \mathrm{s}$.

For a typical X-band spectrometer, these values lead to a spin sensitivity of $10^{11} \mathrm{spins} / \mathrm{G}$ or $5 \times 10^{11} \mathrm{spins} / \mathrm{G} \mathrm{cm}^{3}$. At $\mathrm{W}$-band, the typical values are $N_{\text {min }}=2 \times 10^{7}$ spins/G or $10^{11}$ spins $/ \mathrm{G} \mathrm{cm}^{3}$.

These values, however, assume that the sensitivity of the spectrometer is determined by the noise floor of the detector only (e.g., $-160 \mathrm{dBm} / \mathrm{Hz}$ for a Hecooled bolometer mixer). In practice, also noise contributions from the sources may affect the actual sensitivity. It has been shown that amplitude noise from, e.g., a Gunn oscillator, at $90 \mathrm{GHz}(<-150 \mathrm{dBc} / \mathrm{Hz}$ at $1-100 \mathrm{kHz}$ offset $)$ usually does not play a role because of the low $(-20 \mathrm{dBm})$ LO level needed for the bolometer mixer [57]. Phase noise potentially poses a bigger problem. The conversion of phase noise to amplitude noise by a resonator and a reference arm has been described by Smith and Lesurf [106]. This effect can easily dominate the detector noise floor unless the excitation signal from the probe head is properly isolated by cavity coupling and/or polarization decoupling (induction mode), and the reference arms in the bridge are properly balanced [57].

\section{Conclusions and Outlook}

A practical overview of high-frequency EPR instrumentation was provided in this contribution. It has been shown that for up to $150 \mathrm{GHz}$, the conventional waveguide technology can be used, albeit with some limitations. It is to be expected, however, that quasi-optical technology will rapidly take over as the leading strategy in

\footnotetext{
1 These noise factors depend on many instrumental properties and will vary between various types of EPR spectrometers. How this works out in practice is analyzed in more detail in the recent review by Rinard et al. [2].
} 
developing mm-wave EPR bridges. The spectacular developments in $\mathrm{THz}$ technology and radio astronomy provide an increasing number of components that can be used in high-frequency EPR instruments. In addition, mm-wave excitation also has drawn the attention of the NMR community. In the last few years, many projects on high-field (DNP) have been initiated [107]. It is expected that in the near future, technical developments in high-frequency EPR will occur even more rapidly.

Acknowledgments This paper is dedicated to Wolfgang Lubitz in recognition of his truly impressive career in science and his innovative contributions to the field of EPR and ENDOR. I would like to thank Wolfgang for his continuing support and personal involvement in and outside the laboratory. Also, the generous financial support of the Max-Planck Gesellschaft as well as the Deutsche Forschungsgemeinschaft (DFG) is gratefully acknowledged.

Open Access This article is distributed under the terms of the Creative Commons Attribution Noncommercial License which permits any noncommercial use, distribution, and reproduction in any medium, provided the original author(s) and source are credited.

\section{References}

1. C.P. Poole, Electron Spin Resonance (John Wiley, New York, 1983)

2. G.A. Rinard, S.S. Eaton, G.R. Eaton, C.P. Poole, Farrah H.A, Sensitivity, in Handbook of Electron Spin Resonance, vol 2 ed. by C.P. Poole, H.A. Farrah. (Spinger, New York, 1999) pp. 1-24

3. K.A. Earle, B. Dzikovski, W. Hofbauer, J.K. Moscicki, J.H. Freed, Magn. Reson. Chem. 43, S256S266 (2005)

4. J.H. Freed, Annu. Rev. Phys. Chem. 51, 655-689 (2000)

5. K. Mobius, A. Savitsky, High-field EPR Spectroscopy on Proteins and Their Model Systems (Royal Society of Chemistry, Cambridge, 2009)

6. J.B. Mock, Rev. Sci. Instrum. 31, 551-555 (1960)

7. P.R. Elliston, G.J. Troup, D.R. Hutton, J. Sci. Instrum. 40, 586 (1963)

8. E. Slade, D. Ingram, Proc. Roy. Soc. Lond. A 312, 85-98 (1969)

9. H. Vandenboom, Rev. Sci. Instrum. 40, 550 (1969)

10. I. Amity, Rev. Sci. Instrum. 41, 1492 (1970)

11. H.C. Box, H.G. Freund, K.T. Lilga, E.E. Budzinsk, J. Phys. Chem. 74, 40 (1970)

12. Y. Alpert, Y. Couder, J. Tuchendl, H. Thome, Biochim. Biophys. Acta 322, 34-37 (1973)

13. O.Y. Grinberg, A.A. Dubinskii, The Early Years, in Very High-Frequency $(V H F)$ ESR/EPR, vol. 22, ed. by O.Y. Grinberg, L.J. Berliner (Kluwer/Plenum Publishers, New York, 2004), pp. 1-18

14. Y.S. Lebedev, Very High-field EPR, in Foundations of Modern EPR, ed. by S. Eaton, K.M. Salikhov (World Scientific, Singapore, 1998), pp. 731-740

15. Y.S. Lebedev, Very High-field EPR, in Modern Pulsed and Continuous Wave Electron Spin Resonance, ed. by L. Kevan, M.K. Bowman (John Wiley, New York, 1990), p. 365

16. Y.S. Lebedev, Appl. Magn. Reson. 7, 339-362 (1994)

17. A.A. Galkin, O.Y. Grinberg, A.A. Dubinskii, N.N. Kabdin, V.N. Krymov, V.I. Kurochkin, Y.S. Lebedev, L.F. Oranskii, V.F. Shuvalov, Instrum. Exp. Tech. 20, 1229 (1977)

18. W.B. Lynch, K.A. Earle, J.H. Freed, Rev. Sci. Instrum. 59, 1345-1351 (1988)

19. R.T. Weber, J.A.J.M. Disselhorst, L.J. Prevo, J. Schmidt, W.T. Wenckebach, J. Magn. Reson. 81, 129-144 (1989)

20. J.A.J.M. Disselhorst, H. Vandermeer, O.G. Poluektov, J. Schmidt, J. Magn. Reson. Ser. A 115, 183188 (1995)

21. J. Allgeier, J.A.J.M. Disselhorst, R.T. Weber, W.T. Wenckebach, J. Schmidt, High-Frequency Pulsed Electron Spin Resonance, in Modern Pulsed and Continuous Wave Electron Spin Resonance, ed. by L. Kevan, M.K. Bowman (Wiley, New York, 1990), pp. 267-283

22. O. Burghaus, M. Rohrer, T. Gotzinger, M. Plato, K. Mobius, Meas. Sci. Technol. 3, 765-774 (1992)

23. T.F. Prisner, M. Rohrer, K. Mobius, Appl. Magn. Reson. 7, 167-183 (1994) 
24. O. Burghaus, A. Tothkischkat, R. Klette, K. Mobius, J. Magn. Reson. 80, 383-388 (1988)

25. D. Schmalbein, G.G. Maresch, A. Kamlowski, P. Hofer, Appl. Magn. Reson. 16, 185-205 (1999)

26. P. Hofer, A. Kamlowski, G.G. Maresch, D. Schmalbein, R.T. Weber, The Bruker ELEXSYS E600/ $680 \mathrm{~W}$-band Spectrometer Series, in Very High-Frequency (VHF) ESR/EPR, vol. 22, ed. by O.Y. Grinberg, L.J. Berliner (Kluwer/Plenum Publishers, New York, 2004), pp. 401-429

27. P. Carl, R. Heilig, D.C. Maier, P. Hofer, D. Schmalbein, Bruker Spin Rep. 154/155, 35 (2009)

28. I. Gromov, V. Krymov, P. Manikandan, D. Arieli, D. Goldfarb, J. Magn. Reson. 139, 8-17 (1999)

29. R.J. Hulsebosch, I.V. Borovykh, S.V. Paschenko, P. Gast, A.J. Hoff, J. Phys. Chem. B 103, 68156823 (1999)

30. K.V. Lakshmi, M.J. Reifler, G.W. Brudvig, O.G. Poluektov, A.M. Wagner, M.C. Thurnauer, J. Phys. Chem. B 104, 10445-10448 (2000)

31. P. Dorlet, S.A. Seibold, G.T. Babcock, G.J. Gerfen, W.L. Smith, A.L. Tsai, S. Un, Biochemistry 41, 6107-6114 (2002)

32. A. Gunn, M. Brynda, R.D. Britt, Abstr. Pap. Am. Chem. Soc. 229, 319 (2005)

33. T.A. Stich, S. Lahiri, G. Yeagle, M. Dicus, M. Brynda, A. Gunn, C. Aznar, V.J. Derose, R.D. Britt, Appl. Magn. Reson. 31, 321-341 (2007)

34. G.M. Smith, J.C.G. Lesurf, R.H. Mitchell, P.C. Riedi, Rev. Sci. Instrum. 69, 3924-3937 (1998)

35. K.A. Earle, D.S. Tipikin, J.H. Freed, Rev. Sci. Instrum. 67, 2502-2513 (1996)

36. W. Hofbauer, K.A. Earle, C.R. Dunnam, J.K. Moscicki, J.H. Freed, Rev. Sci. Instrum. 75, 11941208 (2004)

37. P.A.S. Cruickshank, D.R. Bolton, D.A. Robertson, D.J. Keeble, G.M. Smith, in Conference Digest of the2004 Joint 29th International Conference on Infrared and Millimeter Waves and 12th International Conference onTerahertz Electronics (2004), pp. 171-172

38. M. Rohrer, O. Brugmann, B. Kinzer, T.F. Prisner, Appl. Magn. Reson. 21, 257-274 (2001)

39. H. Blok, J.A.J.M. Disselhorst, S.B. Orlinskii, J. Schmidt, J. Magn. Reson. 166, 92-99 (2004)

40. M.R. Fuchs, T.F. Prisner, K. Mobius, Rev. Sci. Instrum. 70, 3681-3683 (1999)

41. G.W. Morley, L.C. Brunel, J. van Tol, Rev. Sci. Instrum. 79 (2008)

42. E. Reijerse, P.P. Schmidt, G. Klihm, W. Lubitz, Appl. Magn. Reson. 31, 611-626 (2007)

43. A.L. Barra, L.C. Brunel, J.B. Robert, Chem. Phys. Lett. 165, 107-109 (1990)

44. L.C. Brunel, A.L. Barra, G. Martinez, Physica B 204, 298-302 (1995)

45. A.K. Hassan, L.A. Pardi, J. Krzystek, A. Sienkiewicz, P. Goy, M. Rohrer, L.C. Brunel, J. Magn. Reson. 142, 300-312 (2000)

46. S.A. Zvyagin, J. Krzystek, P.H.M. van Loosdrecht, G. Dhalenne, A. Revcolevschi, Physica B 346, 1-5 (2004)

47. J. van Slageren, S. Vongtragool, B. Gorshunov, A.A. Mukhin, N. Karl, J. Krzystek, J. Telser, A. Muller, C. Sangregorio, D. Gatteschi, M. Dressel, Phys. Chem. Chem. Phys. 5, 3837-3843 (2003)

48. J. van Slageren, S. Vongtragool, B. Gorshunov, A.A. Mukhin, M. Dressel, J. Magn. Magn. Mater. 272, E765-E767 (2004)

49. C. Golze, A. Alfonsov, R. Klingeler, B. Buchner, V. Kataev, C. Mennerich, H. Klauss, M. Goiran, J. Broto, H. Rakoto, S. Demeshko, G. Leibeling, F. Meyer, Phys. Rev. B 73(22), 224403-224408 (2006)

50. L.R. Becerra, G.J. Gerfen, B.F. Bellew, J.A. Bryant, D.A. Hall, S.J. Inati, R.T. Weber, S. Un, T.F. Prisner, A.E. Mcdermott, K.W. Fishbein, K.E. Kreischer, R.J. Temkin, D.J. Singel, R.G. Griffin, J. Magn. Reson. Ser. A 117, 28-40 (1995)

51. M.J. Nilges, A.I. Smirnov, R.B. Clarkson, R.L. Belford, Appl. Magn. Reson. 16, 167-183 (1999)

52. H.Y. Xu, Y.W. Duan, J.L. Hesler, T.W. Crowe, R.M. Weikle, IEEE Trans. Microw. Theory 54, 3648-3653 (2006)

53. T.W. Crowe, J.L. Hesler, D.W. Porterfield, D.S. Kurtz, K. Hui, Joint 32nd International Conference on Infrared and Millimeter Waves and 15th International Conference on Terahertz Electronics, vols 1 and 2 (2007) pp. 607-608

54. H.Y. Xu, G.S. Schoenthal, J.L. Hesler, T.W. Crowe, R.M. Weikle, IEEE Trans. Microw. Theory 55, 648-655 (2007)

55. Q. Xiao, J.L. Hesler, T.W. Crowe, B.S. Deaver, R.M. Weikle, IEEE Microw. Wirel. Co. 17, 241243 (2007)

56. D.R. Bolton, P.S. Cruickshank, D.A. Robertson, G.M. Smith, Electron. Lett. 43, 346-348 (2007)

57. E.J. Reijerse, P.J. van Dam, A.A.K. Klaassen, W.R. Hagen, P.J.M. van Bentum, G.M. Smith, Appl. Magn. Reson. 14, 153-167 (1998)

58. V. Weis, M. Bennati, M. Rosay, J.A. Bryant, R.G. Griffin, J. Magn. Reson. 140, 293-299 (1999) 
59. D.A. Robertson, D.R. Bolton, P.A.S. Cruickshank, G.M. Smith, Joint 32nd International Conference on Infrared and Millimeter Waves and 15th International Conference on Terahertz Electronics, vols 1 and 2 (2007) pp. 517-518

60. P.A.S. Cruickshank, D.R. Bolton, D.A. Robertson, R.J. Wylde, G.M. Smith, 2007 Joint 32nd International Conference on Infrared and Millimeter Waves and 15th International Conference on Terahertz Electronics, vols 1 and 2, (2007) pp. 78-80

61. C. Kutter, H.P. Moll, J. Vantol, H. Zuckermann, J.C. Maan, P. Wyder, Phys. Rev. Lett. 74, 29252928 (1995)

62. C. Kutter, H.P. Moll, J. Vantol, H. Zuckermann, P. Wyder, Infrared Phys. Techn. 36, 245-249 (1995)

63. H.P. Moll, C. Kutter, J. van Tol, H. Zuckerman, P. Wyder, J. Magn. Reson. 137, 46-58 (1999)

64. D.E. Wortman, R.P. Leavitt, The Orotron, in Infrared and Millimeter Waves vol 7: Coherent Sources and Applications, Part II, ed. by K.J. Button (Academic Press, New York, 1983), pp. 321375

65. K. Mizuno, S. Ono, The Ledatron, in Infrared and Millimeter Waves vol 1: Sources of Radiation, ed. by K.J. Button (Academic Press, New York, 1979), pp. 213-233

66. G. Bogomolov, F. Rusin, V. Kushch, Radio engineering and electronic physics. Radiotekhnika i Elektronika 15(4), 730-731 (1970)

67. F. Rusin, G. Bogomolov, Proc. IEEE 57(4), 720-722 (1969)

68. Y.A. Grishin, M.R. Fuchs, A. Schnegg, A.A. Dubinskii, B.S. Dumesh, F.S. Rusin, V.L. Bratman, K. Mobius, Rev. Sci. Instrum. 75, 2926-2936 (2004)

69. S. Takahashi, D.G. Allen, J. Seifter, G. Ramian, M.S. Sherwin, L.C. Brunel, J. van Tol, Infrared Phys. Techn. 51, 426-428 (2008)

70. D.R. Bolton, D.A. Robertson, G.M. Smith, IRMMW-THz2005: the Joint 30th International Conference on Infrared and Millimeter Waves and 13th International Conference on Terahertz Electronics, vols 1 and 2 (2005) pp. 74-75

71. C.A. Saylor, H. van Tol, J. Krzystek, G. Smith, R. Wylde, L.C. Brunel, in Multifrequency High-field Quasi Optic Homodyne EMR Spectrometer (2000) American Physical Society, Annual March Meeting, March 20-24, 2000 Minneapolis, MN, abstract \#S36.191

72. F. Muller, M.A. Hopkins, N. Coron, M. Grynberg, L.C. Brunel, G. Martinez, Rev. Sci. Instrum. 60, 3681-3684 (1989)

73. L.C. Brunel, J. van Tol, A. Angerhofer, S. Hill, J. Krzystek, A.L. Maniero, Modern Developments and Prospects in Multi-Frequency High-Field EMR, in Very High-Frequency (VHF) ESR/EPR, vol. 22, ed. by O.Y. Grinberg, L.J. Berliner (Kluwer/Plenum Publishers, New York, 2004), pp. 465-538

74. D. Goldfarb, Y. Lipkin, A. Potapov, Y. Gorodetsky, B. Epel, A.M. Raitsimring, M. Radoul, I. Kaminker, J. Magn. Reson. 194, 8-15 (2008)

75. H.J. Vandermeer, J.A.J.M. Disselhorst, J. Allgeier, J. Schmidt, W.T. Wenckebach, Meas. Sci. Technol. 1, 396-400 (1990)

76. K.A. Earle, J.H. Freed, in ESR Spectrometer at $250 \mathrm{GHz}$, Millimeter and Submillimeter Waves II (1995) pp. 86-97

77. J.P. Barnes, J.H. Freed, Rev. Sci. Instrum. 68, 2838-2846 (1997)

78. J.P. Barnes, J.H. Freed, Rev. Sci. Instrum. 69, 3022-3027 (1998)

79. K. Mobius, A. Savitsky, A. Schnegg, M. Plato, M. Fuchs, Phys. Chem. Chem. Phys. 7, 19-42 (2005)

80. A. Schnegg, A.A. Dubinskii, M.R. Fuchs, Y.A. Grishin, E.P. Kirilina, W. Lubitz, M. Plato, A. Savitsky, K. Mobius, Appl. Magn. Reson. 31, 59-98 (2007)

81. J. van Tol, L.C. Brunel, R.J. Wylde, Rev. Sci. Instrum. 76 (2005)

82. H. Blok, J.A.J.M. Disselhorst, H. van der Meer, S.B. Orlinskii, J. Schmidt, J. Magn. Reson. 173, 4953 (2005)

83. W. Wang, R.L. Belford, R.B. Clarkson, P.H. Davis, J. Forrer, M.J. Nilges, M.D. Timken, T. Walczak, M.C. Thurnauer, J.R. Norris, A.L. Morris, Y. Zhang, Appl. Magn. Reson. 6, 195-215 (1994)

84. J.S. Hyde, W. Froncisz, J.W. Sidabras, T.G. Camenisch, J.R. Anderson, R.A. Strangeway, J. Magn. Reson. 185, 259-263 (2007)

85. R.R. Mett, J.W. Sidabras, J.S. Hyde, Appl. Magn. Reson. 31, 573-589 (2007)

86. J.W. Sidabras, R.R. Mett, W. Froncisz, T.G. Camenisch, J.R. Anderson, J.S. Hyde, Rev. Sci. Instrum. 78 (2007) 
87. G. Annino, M. Cassettari, I. Longo, M. Martinelli, P.J.M. van Bentum, E. Van der Horst, Rev. Sci. Instrum. 70, 1787-1793 (1999)

88. G. Annino, M. Cassettari, I. Longo, M. Martinelli, Appl. Magn. Reson. 16, 45-62 (1999)

89. G. Annino, M. Cassettari, M. Fittipaldi, I. Longo, M. Martinelli, C.A. Massa, L.A. Pardi, J. Magn. Reson. 143, 88-94 (2000)

90. G. Annino, M. Cassettari, M. Martinelli, P.J.M. van Bentum, Rev. Sci. Instrum. 74, 4536-4538 (2003)

91. G. Annino, M. Cassettari, M. Martinelli, P.J.M. van Bentum, Appl. Magn. Reson. 24, 157-175 (2003)

92. G. Annino, M. Cassettari, M. Martinelli, Appl. Magn. Reson. 26, 447-456 (2004)

93. G. Annino, M. Cassettari, M. Martinelli, Rev. Sci. Instrum. 76 (2005)

94. G. Annino, M. Cassettari, M. Fittipaldi, M. Martinelli, J. Magn. Reson. 176, 37-46 (2005)

95. A.I. Smirnov, T.I. Smirnova, R.L. MacArthur, J.A. Good, R. Hall, Rev. Sci. Instrum. 77 (2006)

96. P.J.M. van Bentum, J.C. Maan, J.W.M. Van Os, A.P.M. Kentgens, Chem. Phys. Lett. 376, 338-345 (2003)

97. A.Y. Bresgunov, A.A. Dubinskii, V. Krymov, Y.G. Petrov, O.G. Poluektov, Y.S. Lebedev, Appl. Magn. Reson. 2, 715-728 (1991)

98. B. Nafradi, R. Gaal, A. Sienkiewicz, T. Feher, L. Forro, J. Magn. Reson. 195, 206-210 (2008)

99. B. Nafradi, R. Gaal, T. Feher, L. Forro, J. Magn. Reson. 192, 265-268 (2008)

100. H. Brutlach, E. Bordignon, L. Urban, J.P. Klare, H.J. Reyher, M. Engelhard, H.J. Steinhoff, Appl. Magn. Reson. 30, 359-372 (2006)

101. G. Feher, in Sensitivity in Microwave Paramagnetic Resonance Absorption Techniques. Bell telephone system; Technical Publication (1956)

102. G.A. Rinard, R.W. Quine, S.S. Eaton, G.R. Eaton, J. Magn. Reson. 154, 80-84 (2002)

103. G.A. Rinard, R.W. Quine, S. Eaton, G.R. Eaton, J. Magn. Reson. 156, 113-121 (2002)

104. G.A. Rinard, R.W. Quine, J.R. Harbridge, R.T. Song, G.R. Eaton, S.S. Eaton, J. Magn. Reson. 140, 218-227 (1999)

105. J.A. Weil, J.R. Bolton, Electron Paramagnetic Resonance: Elementary Theory and Practical Applications (Wiley, New York, 2007)

106. G.M. Smith, J.C.G. Lesurf, IEEE T. Microw. Theory 39, 2229-2236 (1991)

107. V.P. Denysenkov, M.J. Prandolini, A. Krahn, M. Gafurov, B. Endeward, T.F. Prisner, Appl. Magn. Reson. 34, 289-299 (2008) 